University of Nebraska - Lincoln

DigitalCommons@University of Nebraska - Lincoln

Edward Schmidt Publications

Research Papers in Physics and Astronomy

August 1987

\title{
THE BINARY CEPHEID DL CAS AND THE OPEN CLUSTER NGC 129
}

Hugh C. Harris

U.S. Naval Observatory, Flagstaff, Arizona

Douglas L. Welch

Dominion Astrophysical Observatory, Herzberg Institute of Astrophysics, Victoria, British Columbia, Canada

Robert P. Kraft

Lick Observatory, University of California, Santa Cruz, California

Edward G. Schmidt

University of Nebraska-Lincoln, eschmidt1@unl.edu

Follow this and additional works at: https://digitalcommons.unl.edu/physicsschmidt

Part of the Physics Commons

Harris, Hugh C.; Welch, Douglas L.; Kraft, Robert P.; and Schmidt, Edward G., "THE BINARY CEPHEID DL CAS AND THE OPEN CLUSTER NGC 129" (1987). Edward Schmidt Publications. 22.

https://digitalcommons.unl.edu/physicsschmidt/22

This Article is brought to you for free and open access by the Research Papers in Physics and Astronomy at DigitalCommons@University of Nebraska - Lincoln. It has been accepted for inclusion in Edward Schmidt Publications by an authorized administrator of DigitalCommons@University of Nebraska - Lincoln. 
THE BINARY CEPHEID DL CAS AND THE OPEN CLUSTER NGC 129

\author{
HUGH C. HaRris \\ U.S. Naval Observatory, P. O. Box 1149, Flagstaff, Arizona 86002 \\ Douglas L. WeLCH \\ Dominion Astrophysical Observatory, Herzberg Institute of Astrophysics, 5071 W. Saanich Road, Victoria, British Columbia V8X 4M6, Canada \\ ROBERT P. KRAFT \\ Lick Observatory, University of California, Santa Cruz, California 95064
}

EDWARD G. SCHMIDT ${ }^{\text {a) }}$

Department of Physics and Astronomy, University of Nebraska, Lincoln, Nebraska 68588

Received 26 March 1987; revised 24 April 1987

\begin{abstract}
The Cepheid DL Cas and the nonvariable supergiant Star A are confirmed as members of NGC 129, and both are found to be spectroscopic binaries with orbital periods of a few years. Periods and orbits are determined for both stars. Star A is apparently rotating; tidal interaction with its companion is discussed and then rejected as the probable cause of the rotation. The membership of other stars and the velocity of the cluster are redetermined.
\end{abstract}

\section{INTRODUCTION}

The open cluster NGC 129 is noteworthy because it contains in its field as possible members the Cepheid DL Cas and three other supergiants. Study of supergiants that are members of open clusters is important both for our understanding of the evolutionary tracks of supergiants and for our calibration of Cepheid luminosities and distances (e.g., Schmidt 1976; Becker et al. 1977; G. Harris 1980). Occasionally, supergiants have binary companions, and determination of their properties can provide further constraints on the evolutionary models for supergiants. Constraints on the masses of Cepheids in binary systems are of particular importance for our understanding of the evolution of $\mathrm{Ce}$ pheids.

The membership of DL Cas in NGC 129 was proposed by van den Bergh (1957) and Kraft (1957). Kraft (1958) concluded from velocities and spectral classification that DL Cas, and probably all three supergiants (stars A, AA, and $\mathrm{AB}$, classified F5 Ib, K2 Ib, and M0 Ib, respectively), were members. Arp, Sandage, and Stephens (1959) gave photometry of the cluster and designations (and a finding chart) for these stars, agreeing that at least DL Cas and Star A were members. Lenham and Franz (1961) and Frolov (1975) measured proper motions in the cluster. Both studies concluded that DL Cas was a highly probable member, while Star AB must be a field star, but the two studies disagreed regarding the motions of some of the other bright stars. More recently, Schmidt (1980) used four-color and $\mathrm{H} \beta$ photometry to determine photometric membership and reddenings for many of the main-sequence stars. Finally, Eggen (1983) observed several cluster members, including Star A, which he found not to vary photometrically over ten nights. In order to follow up Kraft's (1958) pioneering study of the cluster velocities, and to obtain more extensive and accurate velocities to improve the discrimination regarding cluster

\footnotetext{
a) Visiting Astronomer, Kitt Peak National Observatory, National Optical Astronomy Observatories, which is operated by AURA, Inc., under contract with the National Science Foundation.
}

membership, this paper reports new velocities in NGC 129, with several surprising results.

\section{VELOCITIES OF STARS IN NGC 129}

Two new sources for velocities have been used in this study. First, velocities of the late-type stars (type F and later) near NGC 129 have been measured with the radial-velocity spectrometer on the $1.2 \mathrm{~m}$ telescope at the Dominion Astrophysical Observatory (Fletcher et al. 1982). These data are listed in Table $\mathrm{I}$. Velocities from this instrument are reduced to the IAU system, and generally have an accuracy near $0.4 \mathrm{~km} \mathrm{~s}^{-1}$ (and better than $0.3 \mathrm{~km} \mathrm{~s}^{-1}$ for observations made in the 1986-1987 observing season). However, the accuracy may be closer to $1 \mathrm{~km} \mathrm{~s}^{-1}$ for Star $A$ and for DL Cas because of line broadening or line asymmetries. Second, nine spectra of seven B stars have been measured, taken in 1976 and 1979 with the $2.1 \mathrm{~m}$ telescope at Kitt Peak National Observatory ( $10 \AA \mathrm{mm}^{-1}$, image-tube spectrograph, resolution $1 \AA$ ) as part of Schmidt's (1978) study of the helium abundances in NGC 129 . Usually, only two hydrogen lines were measured (and the interstellar $\mathrm{Ca} \mathrm{H}$ and $\mathrm{K}$ lines as a check on the stability of the velocities), because the helium lines were too broad and weak to give reliable velocities. Therefore, the accuracy of these velocities is estimated to be $10-15 \mathrm{~km} \mathrm{~s}^{-1}$. (An exception is Star C, which is evolved and has sharper Balmer lines than the main-sequence stars. The error in its velocity is probably between 5 and $10 \mathrm{~km} \mathrm{~s}^{-1}$.) The photographic data are listed in Table II.

The present velocities are in good agreement with Kraft's (1958) original study, except for an apparently constant systematic shift. Omitting DL Cas and Star A because of their variable velocities (discussed in Secs. III and IV), the five stars in common to the two sets of data have differences (in the sense this paper minus Kraft) of $-9,-6,-8,-10$, and $-22 \mathrm{~km} \mathrm{~s}^{-1}$, respectively. The most discrepant is Star $\mathrm{C}$ : its difference is significant and indicates that its velocity also may be variable. The best-determined velocities are for the three later-type stars; they suggest that a zero-point error of $+9 \mathrm{~km} \mathrm{~s}^{-1}$ is present for unknown reasons in the earlier 
TABLE I. Velocities measured with the radial-velocity spectrometer.

\begin{tabular}{|c|c|c|c|c|c|c|c|}
\hline $\begin{array}{c}\text { JD } \\
(2400000+)\end{array}$ & $\begin{array}{c}\mathrm{V} \\
\left(\mathrm{km} \mathrm{s}^{-1}\right.\end{array}$ & $\begin{array}{c}\text { Phase } \\
\text { Orb. Pul. }\end{array}$ & $\begin{array}{c}\text { JD } \\
(2400000+)\end{array}$ & $\begin{array}{c}\mathrm{V} \\
\left(\mathrm{km} \mathrm{s}^{-1}\right)\end{array}$ & $\begin{array}{l}\text { Phase } \\
\text { Orb. }\end{array}$ & $\begin{array}{c}\text { JD } \\
(2400000+)\end{array}$ & $\begin{array}{c}\mathrm{V} \\
\left(\mathrm{km} \mathrm{s}^{-1}\right)\end{array}$ \\
\hline \multicolumn{3}{|c|}{ DL Cas } & \multicolumn{3}{|c|}{ Star A } & \multicolumn{2}{|c|}{ Star AA } \\
\hline 44615.836 & -49.43 & 0.3090 .419 & 44586.762 & -51.43 & 0.283 & 44586.730 & -31.43 \\
\hline 45680.675 & -21.33 & 0.8570 .513 & 44615.820 & -54.33 & 0.295 & 44615.844 & -34.33 \\
\hline 45716.766 & -36.49 & 0.9090 .024 & 44675.652 & -50.29 & 0.320 & 44675.637 & -31.89 \\
\hline 45934.968 & -47.93 & 0.2260 .297 & 44680.648 & -50.43 & 0.322 & 44680.660 & -32.23 \\
\hline 46019.828 & -58.52 & $0.350 \quad 0.904$ & 44696.660 & -49.43 & 0.328 & 44696.668 & -32.33 \\
\hline 46028.660 & -63.65 & 0.3620 .008 & 44824.977 & -47.01 & 0.381 & 44824.980 & -32.15 \\
\hline 46035.664 & -54.26 & 0.3730 .884 & 44830.930 & -47.35 & 0.383 & 44830.938 & -32.68 \\
\hline 46037.730 & -58.15 & 0.3760 .142 & 44844.961 & -46.33 & 0.389 & $\mathbf{4 4 8 4 4 . 9 6 9}$ & -32.70 \\
\hline 46039.609 & -50.48 & $\begin{array}{lll}0.378 & 0.377\end{array}$ & 44938.883 & -43.58 & 0.428 & 44938.895 & -32.65 \\
\hline 46047.664 & -51.38 & 0.3900 .383 & 45009.734 & -42.44 & 0.457 & 45009.746 & -32.33 \\
\hline 46053.570 & -59.37 & 0.3990 .122 & 45047.672 & -41.02 & 0.472 & 45198.980 & -32.96 \\
\hline 46067.738 & -53.68 & 0.4190 .892 & 45198.977 & -38.02 & 0.534 & $\mathbf{4 5 2 5 5 . 7 7 0}$ & -33.45 \\
\hline 46088.625 & -43.67 & 0.4500 .503 & 45255.770 & -36.08 & 0.558 & 45582.969 & -32.38 \\
\hline 46093.750 & -58.26 & 0.4570 .144 & 45582.957 & -25.88 & 0.692 & 46603.801 & -29.32 \\
\hline 46100.672 & -61.11 & 0.4670 .009 & 45625.890 & -25.23 & 0.709 & & \\
\hline 46345.890 & -12.06 & 0.8230 .659 & 45958.047 & -24.27 & 0.845 & \multicolumn{2}{|c|}{ Star AB } \\
\hline 46583.918 & -42.95 & 0.1700 .410 & 45991.027 & -22.45 & 0.859 & 46028.680 & -9.91 \\
\hline 46584.949 & -33.00 & 0.1710 .539 & 46035.670 & -26.15 & 0.877 & 46603.806 & -8.87 \\
\hline 46603.786 & -52.35 & 0.1980 .893 & 46037.734 & -22.25 & 0.878 & & \\
\hline 46604.759 & -57.16 & $0.200 \quad 0.015$ & 46039.617 & -23.82 & 0.879 & \multicolumn{2}{|c|}{ Star B } \\
\hline 46605.751 & -52.83 & 0.2010 .139 & 46093.756 & -27.20 & 0.901 & 46019.836 & -27.33 \\
\hline 46617.859 & -28.95 & 0.2190 .652 & 46100.680 & -25.08 & 0.904 & 46028.676 & -26.94 \\
\hline 46620.818 & -58.87 & 0.2230 .022 & 46583.923 & -47.46 & 0.102 & & \\
\hline 46626.770 & -29.76 & 0.2320 .766 & 46603.792 & -46.88 & 0.110 & & \\
\hline 46631.762 & -46.27 & 0.2390 .390 & 46604.767 & -50.40 & 0.110 & & \\
\hline 46641.960 & -28.10 & 0.2540 .665 & 46649.757 & -49.44 & 0.129 & & \\
\hline 46649.735 & -29.67 & 0.2650 .637 & 46710.686 & -52.10 & 0.154 & & \\
\hline 46654.759 & -51.49 & $0.272 \quad 0.265$ & $\mathbf{4 6 7 5 1 . 8 7 0}$ & -51.75 & 0.171 & & \\
\hline 46656.782 & -41.67 & 0.2750 .517 & 46833.641 & -52.85 & 0.204 & & \\
\hline 46657.721 & -32.2 & 0.2770 .635 & 46873.690 & -52.11 & 0.221 & & \\
\hline 46669.972 & -55.5 & 0.2960 .166 & & & & & \\
\hline 46690.742 & -32.8 & 0.3250 .762 & & & & & \\
\hline 46695.786 & -49.8 & 0.3320 .392 & & & & & \\
\hline 46710.678 & -52.96 & 0.3540 .254 & & & & & \\
\hline 46719.691 & -50.67 & 0.3670 .380 & & & & & \\
\hline 46751.758 & -50.76 & 0.4130 .388 & & & & & \\
\hline 46766.705 & -53.11 & 0.4350 .257 & & & & & \\
\hline 46767.672 & -49.48 & 0.4370 .378 & & & & & \\
\hline 46770.686 & -30.69 & 0.4410 .754 & & & & & \\
\hline 46770.970 & -33.98 & 0.4410 .790 & & & & & \\
\hline 46833.626 & -31.74 & 0.5320 .621 & & & & & \\
\hline 46873.682 & -29.67 & 0.5910 .628 & & & & & \\
\hline
\end{tabular}

results. Applying a correction to the earlier results and combining them with the data in Tables I and II, we can estimate cluster membership from the velocities alone. Table III gives a summary of the results. For stars in common, we find good agreement with both the proper-motion memberships determined by Lenham and Franz (1961) and Frolov (1975) and the photometric memberships determined by Schmidt (1980). (Table III lists DL Cas and Star A as cluster members and Star AA as doubtful, anticipating the discussion in Secs. III and IV.) Among the marginal proper-motion members, we find stars $\mathrm{A}$ and 63 to be probable members, while stars S2, S4, and 125 are probable field stars.
The cluster velocity, based on the results in Table III, is approximately $-38 \mathrm{~km} \mathrm{~s}^{-1}$. The velocities of the mainsequence stars are still not very well determined, and do not contribute very much weight to this number. Additional spectra of candidate main-sequence stars are being obtained at DAO to determine membership with greater precision for more stars.

\section{THE CEPHEID DL CAS}

The recent velocities of DL Cas show that it is a spectroscopic binary with a period of slightly less than two years. 
TABLE II. Radial velocities measured photographically.

\begin{tabular}{lccc}
\hline \hline Star & Plate & $\begin{array}{c}\text { JD } \\
(2400000+)\end{array}$ & $\begin{array}{c}\text { V } \\
\left(\mathrm{km} \mathrm{s}^{-1}\right)\end{array}$ \\
\hline AC & 8949 & 44091.852 & -38. \\
AE & 8950 & 44091.975 & $-55 .:$ \\
C & 6422 & 42966.929 & -42. \\
61 & 8951 & 44093.928 & $-50 .:$ \\
63 & 6426 & 42968.960 & -38. \\
& 8951 & 44093.960 & $-50 .:$ \\
105 & 6422 & 42966.957 & -39. \\
& 6424 & 42967.840 & -32. \\
125 & 6426 & 42968.868 & -68. \\
\hline
\end{tabular}

Observations have been made near maximum positive velocity in late 1983 and 1985. Fitting the present data (from Table I) for the orbital elements including the period gives a period of $689 \pm 6$ days. Kraft (1958) observed DL Cas on one observing run in October 1957, from JD 2436112 through 2436122 . His velocities show it was then near maximum positive velocity, and this fact provides a slightly tighter constraint on the period. Combining the two epochs of maximum velocity (JD 2436117 in 1957 and JD 2445747 one month after the observation in January 1984), we find the orbital period to be $688.0 \pm 4$ days, with 14 cycles separating the two epochs. Alternative periods, such as 741 days (with 13 cycles separating the two epochs) or 642 days ( 15 cycles), are ruled out by the combined data in Table I. Therefore, we adopt a period of 688.0 days for the final orbit solution. Twenty additional velocities observed by Moffett and Barnes in 1979, 1980, and 1981 were recently communicated to us in advance of publication (Barnes 1986; Barnes $e t$ al. 1987) and are consistent with a period of 688 days.

The velocity curves for DL Cas are plotted in Fig. 1. Kraft's (1958) photographic velocities were not used in the orbit solution, but they have been included in the plot after applying a shift of $-9 \mathrm{~km} \mathrm{~s}^{-1}$, as discussed in Sec. II. The pulsation phases were calculated from the ephemerides of the light curve given by Moffett and Barnes (1984):
$P=8.000610$ and $\operatorname{JD}\left(V_{\max }\right)=2437043.910$. (No shift in phase has been applied, and the agreement with the current pulsation ephemerides is surprisingly good.) The pulsation and orbital velocities have each been removed from the plot of the other, and the pulsation curve is defined to have an integral velocity of zero. To fit the data, each curve was fit alternately after removing the other, and three iterations were made. The elements of the orbit are

$$
\begin{aligned}
& P=688.0 \pm 4.4 \text { days, } \\
& \gamma=-38.1 \pm 0.4 \mathrm{~km} \mathrm{~s}^{-1}, \\
& K_{1}=14.0 \pm 0.9 \mathrm{~km} \mathrm{~s}^{-1}, \\
& e=0.28 \pm 0.04 \\
& \omega=26^{\circ} \pm 7^{\circ} \\
& T_{0}=2445779 \pm 15 \mathrm{JD}, \\
& \sigma=0.88 \mathrm{~km} \mathrm{~s}^{-1}, \\
& f(m)=0.172 \pm 0.033 \mathscr{M}_{\odot} .
\end{aligned}
$$

The $\gamma$ velocity agrees with several other probable cluster members, although the values are quite different than found earlier by Kraft. The evidence from velocities, proper motions, spectra, and position near the center of the cluster leaves little doubt that DL Cas is a member of NGC 129.

The amplitude of the orbital velocity curve is quite large, perhaps surprisingly so considering that the binary motion has not been detected until now. The size of the orbit is not unreasonable for a supergiant: $(a \sin i)=1.27 \times 10^{8}$ $\mathrm{km}=0.85 \mathrm{AU}$. The radius of DL Cas is about $3.5 \times 10^{7} \mathrm{~km}$, based on the distance found by Schmidt (1980) and the temperature $(5650 \mathrm{~K})$ found by Harris and Pilachowski (1984). Therefore, DL Cas is now comfortably smaller than the separation of its companion. Because most Cepheids are believed to be making second or later transitions of the instability strip, DL Cas was probably a red supergiant earlier in

\begin{tabular}{|c|c|c|c|c|c|c|c|c|}
\hline \multirow[b]{2}{*}{$\begin{array}{l}\text { Arp } \\
\text { et al. }\end{array}$} & \multicolumn{4}{|c|}{ Star Designation } & \multirow[b]{2}{*}{$\begin{array}{l}\text { Lenham } \\
\text { and Franz }\end{array}$} & \multirow{2}{*}{$\begin{array}{l}\text { Velocity } \\
\left(\mathbf{k m ~ s}^{-1}\right)\end{array}$} & \multirow{2}{*}{$\begin{array}{c}\text { Spectral } \\
\text { Type }\end{array}$} & \multirow{2}{*}{$\begin{array}{c}\text { Cluster } \\
\text { Member? }\end{array}$} \\
\hline & $\begin{array}{c}\text { HD } \\
\text { or } \mathrm{HDE}\end{array}$ & BD & SAO & $\begin{array}{l}\text { Hoag } \\
\text { et al. }\end{array}$ & & & & \\
\hline DL Cas & 236429 & +5965 & 21446 & 4 & 34 & -38.1 & F5-G3Ib & Yes \\
\hline $\mathbf{A}$ & 236433 & +5967 & 21450 & 5 & 38 & -38.0 & F5Ib & Yes \\
\hline $\mathbf{A A}$ & 236449 & +5973 & 21482 & 2 & 62 & -32.6 & $\mathrm{~K} 2 \mathrm{Ib}$ & Doubtful \\
\hline $\mathbf{A B}$ & 236446 & +5970 & 21478 & 3 & 57 & -9.4 & MOIb & No \\
\hline $\mathbf{A C}=\mathbf{S} 1$ & 236452 & $\ldots$ & 21485 & 10 & 63 & -38 & B9V & Yes \\
\hline $\mathbf{A E}$ & ... & ... & $\ldots$ & 22 & 59 & $-55 .:$ & B: & Probably \\
\hline B & 236436 & ... & 21458 & 7 & 42 & -27.1 & F6V & No \\
\hline $\mathbf{C}$ & $\ldots$ & ... & $\ldots$ & 11 & 44 & $-36 .:$ & B6III-IV & Yes \\
\hline 61 & ... & ... & $\ldots$ & ... & 45 & $-50 .:$ & B5V & Yes \\
\hline 63 & ... & $\ldots$ & $\ldots$ & $\ldots$ & 46 & -40 & $\ldots$ & Probably \\
\hline 105 & ... & ... & $\ldots$ & 13 & 39 & -36 & B9V & Yes \\
\hline 125 & $\ldots$ & $\cdots$ & $\ldots$ & 18 & 30 & -68 & B: & No \\
\hline S2 & 236448 & +5971 & 21479 & $\ldots$ & 60 & +5 & AOV & No \\
\hline S3 & 2745 & +5972 & 21480 & $\ldots$ & $\ldots$ & -21 & AOV & No \\
\hline S4 & $\ldots$ & $\ldots$ & $\ldots$ & $\ldots$ & 65 & -12 & B8.5V & No \\
\hline
\end{tabular}
its evolution, and its radius would have been larger then. Whether the maximum radius would exceed the Roche limit has been discussed for other Cepheids and supergiants by several authors (e.g., Plavec 1967; Lloyd Evans 1982; Burki and Mayor 1983). We can estimate the ratio of maximum size to present size from evolutionary tracks (Becker et al.

TABLE III. Summary. 

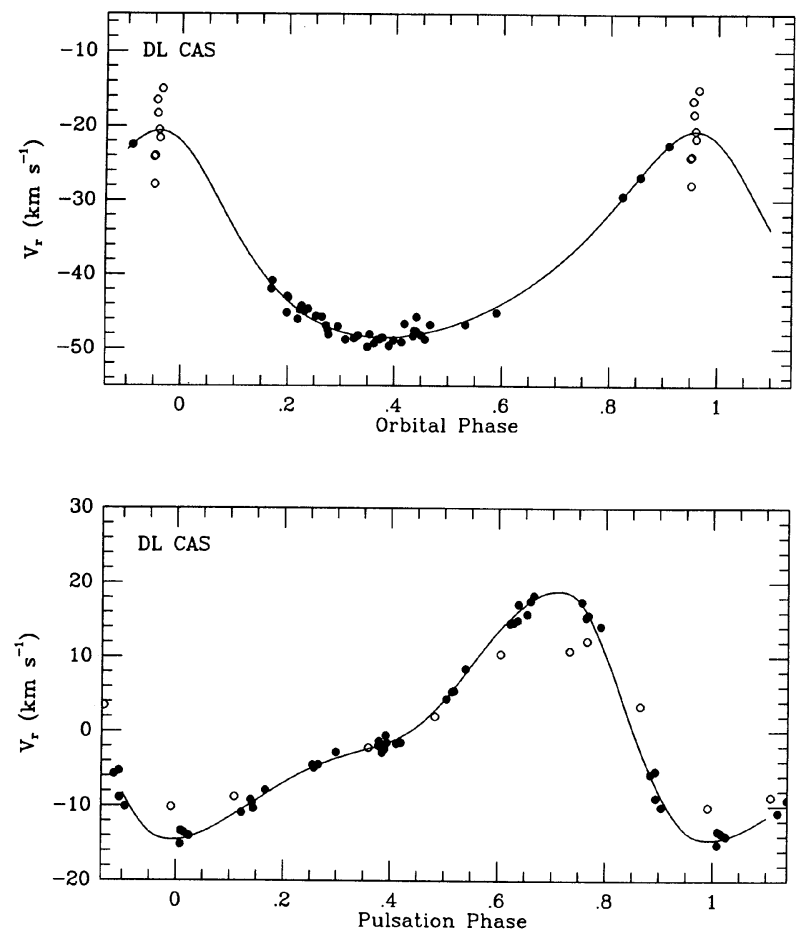

FIG. 1. Velocity curves for DL Cas, showing the orbital velocity (upper panel) and the pulsation velocity (lower panel). The dark circles show data from the DAO radial-velocity spectrometer. The light circles are taken from the photographic data of Kraft (1958). The curves have been fitted to the dark circles only.

1977), but the ratio is quite sensitive to the exact composition and mass of DL Cas, to which crossing of the strip DL Cas is now making, and to the details of the evolutionary models. For reasonable assumptions ( $Y$ near $0.24, Z$ near 0.02 , mass near $8 \mathscr{M}_{\odot}$, second crossing) the ratio is approximately 1.5 to 3 . Therefore, DL Cas could have approached or exceeded its Roche limit during its red supergiant evolution. However, considering that the inclination is unknown (small values of $i$ lead to larger orbits) and that the companion is likely to be less massive, DL Cas has probably evolved to its present state without yet having reached its Roche limit at any time in its evolution.

With a mass function of $0.172 \mathscr{M}_{\odot}$ and a mass for DL Cas of 6 to $9 \mathscr{M}_{\odot}$, the mass of the companion is near $2.5 \mathscr{M}_{\odot}$ for inclinations near $90^{\circ}$, near $3 \mathscr{M}_{\odot}$ for inclinations near $60^{\circ}$, and near $6 \mathscr{M}_{\odot}$ for inclinations near $30^{\circ}$. A mass of 3 to 5 $\mathscr{M}_{\odot}$ corresponds to a main-sequence star of late B or A0 spectral type. A companion this far down the main sequence will be only marginally detectable at best with the various methods proposed for detecting blue companions to $\mathrm{Ce}$ pheids (cf. Madore 1977; Fernie 1980). DL Cas was noted by Madore and Fernie (1980) as having a phase shift suggestive of a blue companion, but has not been identified otherwise as having a companion. An IUE ultraviolet spectrum taken by Evans (1986) shows evidence for a small excess flux in the region of $2500 \AA$. The spectrum is consistent with the presence of a late B main-sequence star, and rules out an early B main-sequence star. Therefore, a main-sequence companion of more than about $6 \mathscr{M}_{\odot}$ (with an inclination less than about $30^{\circ}$ ) can be excluded. A degenerate companion left from a star originally more massive than DL Cas is also possible. Such a companion could be presently less massive or more massive than DL Cas, depending on the orbital inclination.

The pulsation curve for DL Cas from the observations made with the radial-velocity spectrometer (the dark circles in Fig. 1) is quite well defined. The earlier photographic velocities (light circles) indicate a systematically smaller pulsation amplitude. This discrepancy might arise from the greater spectral resolution of the radial-velocity spectrometer. Near the extreme velocities, the line profile is intrinsically asymmetric because different projection factors apply to light coming from different parts of the stellar disk. Because the line profile is partially resolved by the spectrometer, and velocities are determined from the core of the crosscorrelation profile, the spectrometer might observe a larger pulsation amplitude than a spectrograph with lower resolution. A Baade-Wesselink analysis using these or similar data will have to take into account this effect through the projection factor used to convert between observed velocities and pulsation velocities.

\section{THE OTHER SUPERGIANTS}

Star A is a spectroscopic binary with properties similar to DL Cas. Kraft (1958) also observed it near maximum positive velocity. The early and recent epochs of maximum imply a period of $2440 \pm 40$ days, with 4 cycles separating the two epochs. (A period of 1960 days, with 5 cycles separating the two epochs, is ruled out by our observations this season. A period of 3250 days, with 3 cycles between the two epochs, is still possible, but gives systematic residuals from the bestfit orbit for our most recent data, so is not likely to be correct.) The velocity curve is plotted in Fig. 2. Again, Kraft's velocity is included in the figure after applying a shift of -9 $\mathrm{km} \mathrm{s}^{-1}$. The elements of the orbit are

$$
\begin{aligned}
& P=2440 \pm 40 \text { days, } \\
& \gamma=-38.0 \pm 0.2 \mathrm{~km} \mathrm{~s}^{-1} \\
& K_{1}=15.1 \pm 0.3 \mathrm{~km} \mathrm{~s}^{-1} \\
& e=0.14 \pm 0.02 \\
& \omega=84^{\circ} \pm 12^{\circ} \\
& T_{0}=2446335 \pm 87 \mathrm{JD}, \\
& \sigma=1.16 \mathrm{~km} \mathrm{~s}^{-1}, \\
& f(m)=0.848 \pm 0.053 \mathscr{M}_{\odot} .
\end{aligned}
$$

As with DL Cas, the evidence from velocities, proper motions, spectra, and position near the center of the cluster leave little doubt that Star A is also a member of NGC 129.

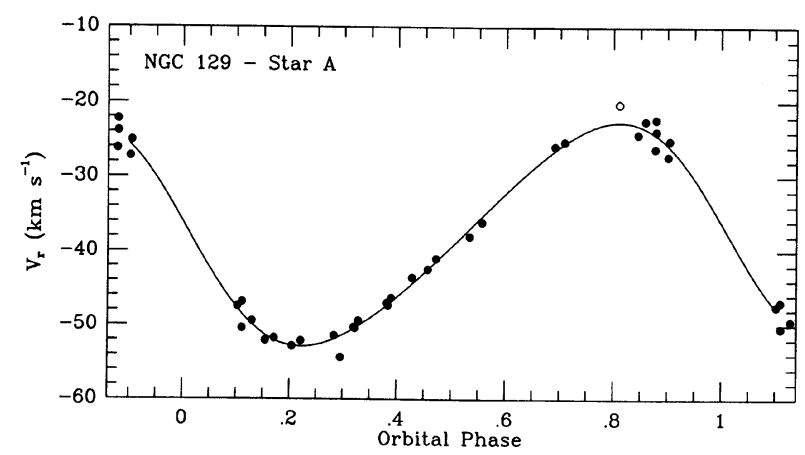

FIG. 2. Orbital-velocity curve of Star A. The symbols are the same as in Fig. 1. 
Star A exhibits line broadening larger than most FIb supergiants (Abt 1958; Harris 1985). One example of a crosscorrelation profile from the DAO radial-velocity spectrometer used to derive this result is shown in Fig. 3. The symmetric, broad profile for this Ib supergiant with an otherwise normal spectrum suggests that rotation, possibly in synchronism with the orbital motion, is the source of the line broadening. We assume so for the following discussion. The projected rotation velocity derived from the cross-correlation profile is $19 \pm 6 \mathrm{~km} \mathrm{~s}^{-1}$. The quoted error is the formal error in the fit to the profile in Fig. 3. Possible systematic errors in the result, arising from the various factors that might affect a cross-correlation profile, are expected to be relatively smaller (Benz and Mayor 1981). Star A has a temperature about $6600 \mathrm{~K}$, based on its colors (bluer than DL Cas in $B-V, T_{1}-T_{2}$, and $b-y$ ) and spectral type (F5 compared to F5-G3 for DL Cas), and is slightly brighter than DL Cas. (The temperature difference between Star A and DL Cas is uncertain partly because the two stars may have different foreground reddening, as discussed by Schmidt (1980). We have used Schmidt's reddening estimates in this discussion.) Therefore, Star $\mathbf{A}$ has a radius about $30 \%$ smaller than DL Cas, or about $2.7 \times 10^{7} \mathrm{~km}$. The rotation period implied by combining this radius with the rotation velocity is $\leqslant 100$ days, where the inequality results from the unknown inclination of the rotation axis, and is a factor of at least 20 smaller than the orbital period. The size of the orbit is $(a \sin i)=5 \times 10^{8} \mathrm{~km}=3 \mathrm{AU}$, larger than the orbit of DL Cas and much larger than the size of Star A. Although the eccentricity of the orbit means that the separation will be smaller at periastron passage than the average separation, the factor $(1-e)$ is not large enough to make much difference. Both the long orbital period and the large semimajor axis imply that no tidal interaction is now occurring between Star $\mathrm{A}$ and its companion.

Nevertheless, tidal spin-up is attractive as a possible explanation for the rotation of Star A. Can tidal effects have played a role in spite of its relatively wide present separation? As a red supergiant, its radius could have become only slightly larger than $10^{8} \mathrm{~km}$, as we calculated for DL Cas in

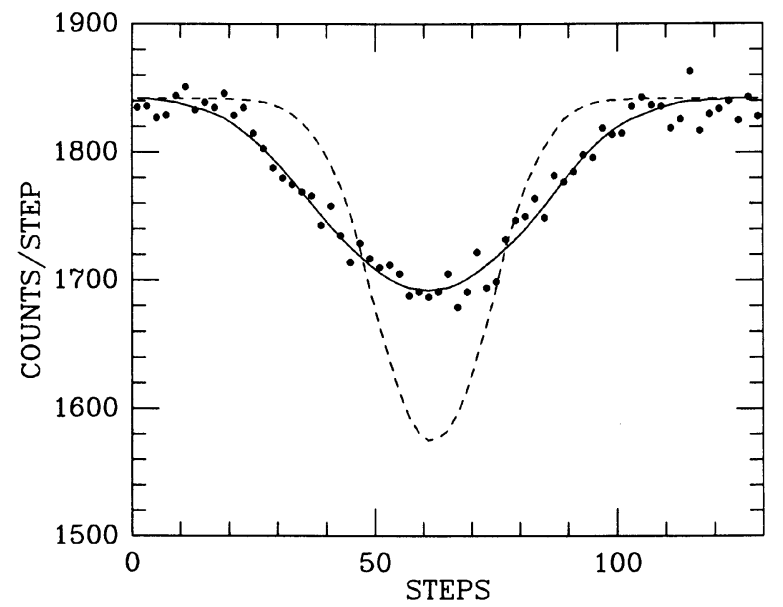

FIG. 3. Example of the cross-correlation profile of Star A, observed on 1982 September 13. The data are binned into two steps per point, with $0.6 \mathrm{~km} \mathrm{~s}^{-1}$ per step. The solid line shows a least-squares fit for line depth, line position, and amount of rotational broadening. The dashed line shows the profile for the sharp-lined star $\gamma$ Aql.
Sec. III. For tidal interaction to occur would then require a companion considerably more massive than Star A. If Star A filled (or nearly filled) its Roche lobe when it was a red supergiant and was then in synchronous rotation, its rotation period could be reduced to the present observed value by spin-up with angular momentum conserved as it contracted. However, this explanation would require Star A to have maintained its rotation during the phase of contraction to its present radius in spite of magnetic braking while cooler than the instability strip and pulsation while transiting the instability strip. These requirements for Star A to achieve and maintain rotation as a supergiant seem, to us, too demanding to be likely.

Some supergiants may be on their way to becoming red supergiants for the first time, and they may still retain their main-sequence angular momentum. This explanation for the rotation of Star A is possible. Luck (1982) proposed this explanation for the high lithium abundances of two other supergiants with detectable rotation. Line broadening indicative of rotation has been detected in $20 \%$ of a sample of $F$, $\mathrm{G}$, and $\mathrm{K}$ supergiants (Harris 1985). However, conventional evolutionary tracks allow only a very small fraction of supergiants now to be making this initial transition to become red supergiants (Becker et al. 1977). Kraft (1966) argued, on the basis of the distribution of linewidths for a smaller sample of supergiants and Cepheids, that essentially no supergiants exhibited at their surface any evidence of their main-sequence rotation, in agreement with evolutionary theory. However, the more complete and precise data now available do not support that conclusion. That some rotating supergiants do have companions may be relevant. Whether the fraction with companions is higher among rotating supergiants than among nonrotating supergiants is not known. Our present result, that the rotation of Star A is probably not a result of tidal interaction with its companion, suggests that we should not expect tidal spin-up to account for the $20 \%$ of broad-lined supergiants.

Star AA, the K2 Ib supergiant, has a nearly constant velocity over five observing seasons. It may have real variations, but they are less than $2 \mathrm{~km} \mathrm{~s}^{-1}$. Real variations of $0.5-$ $1 \mathrm{~km} \mathrm{~s}^{-1}$ are typical of many supergiants (Harris 1985). There is no evidence in the DAO data of a systematic change of velocity, and Kraft's (1958) velocity is not significantly different, indicating that it is not a spectroscopic binary unless it has a very long period. If it is not a binary, then its velocity is sufficiently different from the velocities of DL Cas and Star A to rule out cluster membership. However, its velocity, position ( 10 arcmin from the cluster center), proper motion, and unusual spectral type all suggest that it may be related to the cluster. Possibly it is a long-period spectroscopic binary and a cluster member, or perhaps it is a former member that has recently escaped.

Star AB, the M0 supergiant, has a velocity of $-9 \mathrm{~km} \mathrm{~s}^{-1}$ from the data of this paper and Kraft (1958), inconsistent with cluster membership. A recent and as yet unconfirmed velocity from Sowell (1987) of $-35.9 \mathrm{~km} \mathrm{~s}^{-1}$ may indicate variable velocity, but cluster membership is still ruled out by its proper motion.

We are grateful to Colin Scarfe and Peter Stetson for obtaining several observations of DL Cas, to Peter Stetson and Chris Morbey for their RVS reduction software, to Murray Fletcher for support of the radial-velocity spectrometer, to 
Nancy Evans for describing her $I U E$ data in advance of publication, and to Kyle Cudworth for excavating the 1957 spectrograms.

Note added in proof: A preprint received from J. C. Mer- milliod, M. Mayor, and G. Burki is in good agreement with our results for the orbit of Star A, the variable velocity of DL Cas, and the nonmembership of Star AB in NGC 129. The authors do not derive an orbit for DL Cas.

\section{REFERENCES}

Abt, H. (1958). Astrophys. J. 127, 658.

Arp, H., Sandage, A., and Stephens, C. (1959). Astrophys. J. 130, 80.

Barnes, T. G. (1986). Private communication.

Barnes, T. G., Moffett, T. J., and Slovak, M. H. (1987). Astrophys. J. Suppl. (submitted).

Becker, S. A., Iben, I., and Tuggle, R. S. (1977). Astrophys. J. 218, 633. Benz, W., and Mayor, M. (1981). Astron. Astrophys. 93, 235.

Burki, G., and Mayor, M. (1983). Astron. Astrophys. 124, 256.

Eggen, O. J. (1983). Astron. J. 88, 361.

Evans, N. R. (1986). Private communication.

Fernie, J. D. (1980). Astron. Astrophys. 87, 227.

Fletcher, J. M., Harris, H. C., McClure, R. D., and Scarfe, C. D. (1982). Publ. Astron. Soc. Pac. 94, 1017.

Frolov, V. N. (1975). Bull. Pulkova Astron. Obs. No. 193, 80.

Harris, G. L. H. (1980). In Star Clusters, IAU Symposium No. 85, edited by J. E. Hesser (Reidel, Dordrecht), p. 195.

Harris, H. C. (1985). In Stellar Radial Velocities, IAU Colloquium No. 68, edited by A. G. D. Philip and D. W. Latham (Davis, Schenectady), p. 283.
Harris, H. C., and Pilachowski, C. A. (1984). Astrophys. J. 282, 655.

Hoag, A. A., Johnson, H. L., Iriarte, B., Mitchell, R. I., Hallam, K. L., and Sharpless, S. (1961). Publ. U.S. Nav. Obs. XVII, 349.

Kraft, R. P. (1957). Astrophys. J. 126, 225.

Kraft, R. P. (1958). Astrophys. J. 128, 161.

Kraft, R. P. (1966). Astrophys. J. 144, 1008.

Lenham, A. P., and Franz, O. G. (1961). Astron. J. 66, 16.

Lloyd Evans, T. (1982). Mon. Not. R. Astron. Soc. 199, 925.

Luck, R. E. (1982). Publ. Astron. Soc. Pac. 94, 811.

Madore, B. F. (1977). Mon. Not. R. Astron. Soc. 178, 505.

Madore, B. F., and Fernie, J. D. (1980). Publ. Astron. Soc. Pac. 92, 315. Moffett, T. J., and Barnes, T. G. (1984). Astrophys. J. Suppl. 55, 389.

Plavec, M. (1967). Bull. Astron. Inst. Czech. 18, 253.

Schmidt, E. G. (1976). Astrophys. J. 203, 466.

Schmidt, E. G. (1978). Astrophys. J. 219, 543.

Schmidt, E. G. (1980). Astron. J. 85, 695.

Sowell, J. R. (1987). Astrophys. J. Suppl. 64, 241. van den Bergh, S. (1957). Astrophys. J. 126, 323. 